\title{
III. Krisenbewältigung im Zeichen der Union nationale von 1926
}

Mit der Union nationale von 1926 überwand das parlamentarische System Frankreichs die akut gewordene Krise überraschend schnell. Poincaré konnte dabei an sein bereits 1922-24 praktiziertes Modell der „überparteilichen“ Regierungsführung anknüpfen, das auf den traditionellen Funktionsweisen des französischen Parlamentarismus aufbaute, diese aber durch eine energische und auf populistische Zustimmung zielende Politik des Président $d u$ conseil ergänzte und in gewisser Weise auch bändigte. Sehr schnell und mit erstaunlichem Erfolg wurden in einem breiten Konsens auch die finanzpolitischen Weichen für eine Stabilisierung des Franc gestellt. Das Ermächtigungsgesetz vom 3. August 1926 war dabei - nach der in der Praxis nie umgesetzten Ermächtigung vom März 1924 und nach der Diskussion um die schließlich verweigerten finanzpolitischen pleins pouvoirs für die Regierung Briand-Caillaux im Juli 1926 - ein dritter und entscheidender Schritt zur Etablierung dieses Instruments der legislativen Funktionsabgabe.

Im folgenden sollen zunächst die Umstände und Hintergründe der erstaunlich reibungslosen Regierungsbildung und der parlamentarischen Durchsetzung des Ermächtigungsgesetzes betrachtet werden. Daran schließt sich ein Ausblick auf die funktionale Konstellation der beiden Jahre bis zu den Kammerwahlen von 1928 an.

\section{Poincarés Regierungsbildung der Union nationale}

Schon kurz nach der Demission des Kabinetts Herriot II am Abend des 21. Juli beauftragte Staatspräsident Doumergue Raymond Poincaré mit der Regierungsbildung1. Daß sich der ehemalige Staats- und Ministerpräsident bereits in Wartestellung befand, dürfte außer Zweifel stehen ${ }^{2}$, denn schon seit Tagen war diese Wendung absehbar gewesen.

Bislang wurde übersehen, daß es hierfür auch eine parlamentarische Initiative gab. Bereits nach dem Sturz der Regierung Briand X hatte sich in der Abgeordnetenkammer auf Initiative von Emile Morinaud (Républicains-socialistes) - eines erfahrenen, in den letzten Jahren aber kaum hervorgetretenen Abgeordneten ${ }^{3}-$ eine "Intergroupe du Salut Public" gebildet, der sich rasch etwa 250 Deputierte, vornehmlich wohl der linken und rechten Mitte, anschlossen 4 . Dieser Vorgang

1 Vgl. allgemein zum folgenden v.a. Bonnefous, Histoire 4, S. 164-166; Keiger, Poincaré, S. 320f.; Berstein, Histoire 2, S. 17 f.; Roth, Poincaré, S. 482-488, insbesondere zur Zusammensetzung des Kabinetts.

2 So Bonnet, Vingt ans, S. 122: „Celui-ci s'y attendait. Son cabinet était déjà presque composé.“ Letzteres dürfte übertrieben sein, v.a. was die Beteiligung von Herriot betrifft.

3 Morinaud war von 1898-1902 Mitglied der Kammer und hier v.a. als Interessenvertreter der algerischen Kolonisten aktiv. Politisch war er zunächst in der Groupe antisémite beheimatet. 1919 zog er erneut in die Kammer ein und schloß sich den Républicains-socialistes an, 1928 wechselte er zu der weiter in der Mitte stehenden Gauche unioniste et sociale. Vgl. Dictionnaire des parlementaires français 7, S. $2522 \mathrm{f}$.

4 Vgl. v.a. Botschaftsbericht-Paris (Hoesch), Tel. Nr. 729, 22. 7. 1926, S. 4; PA AA Berlin, R 70717; sowie Note Jean, 22.7. 1926; AN Paris, F7 12954, Bl. 900. Leicht abweichende Zahlenangaben: 230 bei Hoesch, über 250 im Polizeibericht. - Billard, Parti républicain-socialiste, S. 414, geht 
läßt sich gleichermaßen als Zeichen für die fortbestehende Kraft parlamentarischer Individualität wie für die 1925/26 erfolgte Aufweichung der fraktionellen Konturen bewerten. Ziel der Vereinigung war eine Regierung der nationalen Union, und es ist anzunehmen, daß es auch schon um Poincaré als Kandidaten für eine Ministerpräsidentschaft ging. Sofort nach dem Ende der Regierung Herriot II sprachen Vertreter der Intergroupe bei Doumergue vor. Auch die Unterredung mit Justin de Selves, dem Präsidenten des Senats, und Léo Bouyssou, dem Vizepräsidenten der Abgeordnetenkammer ${ }^{5}$, bestätigte das Staatsoberhaupt in seiner vermutlich ohnehin schon feststehenden Entscheidung für Poincarét.

Im Laufe des 22. Juli, einem Tag von extremer parlamentarischer Spannung und voller währungspolitischer Befürchtungen für den Fall eines Scheiterns der neuen Regierungsbildung ${ }^{7}$, führte Poincaré die entscheidenden Gespräche zur Zusammenstellung seines vierten Kabinetts und zur Absicherung seiner Regierungsmehrheit. Die Zielsetzung einer möglichst breiten Formation wurde gegen Mittag durch eine Delegation der Intergroupe bestärkt, die eine Kammermehrheit von mindestens 300 Abgeordneten zusicherte und um eine proportionale Berücksichtigung der parlamentarischen Linken bei der Kabinettsbildung warb8. Poincaré, der sich immer um einen Brückenschlag nach links bemüht hatte, war hierzu wohl von vornherein entschlossen. Eine sofortige Absage kam aus Gründen der Parteiräson allerdings von dem persönlich durchaus zu einem Regierungseintritt bereiten Paul-Boncour, dem prominentesten Vertreter des rechten SFIO-Flügels ${ }^{9}$. Als sehr unsicher galt weiterhin eine Unterstützung durch die Radicaux, die nach wie vor mit über 130 Deputierten die stärkste Fraktion der Abgeordnetenkammer stellten. Poincaré, so hieß es in einem Bericht der Notes Jean, sei „dans les milieux politiques et de presse" allenfalls als Finanzminister innerhalb eines Kabinetts vorstellbar, das von einem Mitglied des Parti radical geführt werde ${ }^{10}$. Da Herriot und auch Caillaux weitestgehend diskreditiert waren, dachte mancher dabei offenbar an Albert Sarraut, der - nach seinem Ausschluß wegen Unterstützung für Poincarés Ermächtigung von 1924 - inzwischen wieder in die Partei aufgenommen worden war ${ }^{11}$.

Der Schlüssel für den Erfolg Poincarés lag in der Einbeziehung von Herriot, der ein Angebot zur Übernahme des aus radikaler Sicht prestigeträchtigen Unter-

fälschlicherweise davon aus, daß die Intergroupe erst nach Formierung der Union-nationaleRegierung gebildet worden sei.

5 Die Präsidentschaft war nach dem kurzzeitigen Wechsel Herriots in das Amt des Ministerpräsidenten noch vakant.

- Vgl. LT, 23. 7. 1926, S. 3, „Les premières consultations du président de la République“.

7 Vgl. Note Jean, 22. 7. 1926; AN Paris, F7 12954, Bl. 900 f. Demnach herrschten in den couloirs der Abgeordnetenkammer Nervosität und Gerüchte. Für Kreditinstitute und Lebensmittelgeschäfte in Paris galt, offenbar aus Angst vor panikartigen Abhebungen und Hamsterkäufen, verstärkter Polizeischutz.

8 Note Jean, 22. 7. 1926; AN Paris, F7 12954, Bl. 902.

9 Paul-Boncour, Entre deux guerres 2, S. 117 f. Vgl. auch Bonnefous, Histoire 4, S. 165.

10 Note Jean, 22. 7. 1926; AN Paris, F7 12954, Bl. 896. Ähnlich Botschaftsbericht-Paris (Hoesch), Tel. Nr. 736, 23. 7. 1926, S. 1, der davon spricht, „daß unter Radikalsozialisten keine geeignete Person zur Verfügung stand, die bereit gewesen wäre, sich für Präsidentenschaft und Finanzministerium zur Verfügung zu stellen“. PA AA Berlin, R 70717.

1 Der einstige Kolonialminister Poincarés war zwischenzeitlich Botschafter in Konstantinopel gewesen. 
richtsministeriums erhielt ${ }^{12}$. Der gescheiterte Chef des Linkskartells, von Poincaré unter Zeitdruck gesetz.t, beriet sich zunächst mit einer schnell einberufenen Versammlung von Parteifreunden. Als diese zwar keine definitive Entscheidung fällte, aber an der Skepsis gegenüber einer Ministerpräsidentschaft Poincarés festhielt, erklärte sich Herriot auf eigene Faust zum Eintritt in das Kabinett bereit und begründete dies in einem Brief an den Vorsitzenden der radikalen Kammerfraktion, Pierre Cazals, mit seiner nationalen Verantwortung ${ }^{13}$. Der Gewinn für Poincaré war ein doppelter: Zum einen hatte er es geschafft, einen potentiellen Oppositionsführer einzubeziehen und den gegen seine Regierungsbildung formierten Widerständen aus der radikalen Kammerfraktion gleichsam die Spitze zu nehmen. Zum anderen konnte er so mittelfristig die Radicaux in die zu erwartenden unpopulären Maßnahmen einer Währungsstabilisierung einbinden ${ }^{14}$. Herriot aber hatte nach seinem Alleingang vom 17. Juli nun schon zum zweiten Mal in kürzester Zeit wenig Rücksicht auf Partei und Fraktion genommen und damit seinen eigenen, jahrelang verfolgten Kurs mühsamer Konsolidierung und Disziplinierung des Parti radical konterkariert. Die Entwicklung zu einer parteipolitischen Verfestigung des parlamentarischen Lebens hatte damit einen weiteren Rückschlag erlitten.

Mit dem Eintritt von Herriot war das schwierigste Problem der Kabinettsbildung gelöst. Auch die übrige Ministerliste spiegelt Poincarés Bemühen um eine prominente und auch mögliche Gegenspieler einbeziehende Regierungsmannschaft. Louis Barthou, ehemaliger Ministerpräsident und vielfacher Minister, wie Poincaré Senator der Union républicaine, übernahm die kombinierten Ressorts der Justiz, Elsaß-Lothringens und die Vice-Présidence du Conseil. Paul Painlevé, einer der Führer des untergegangenen Linkskartells, kehrte in das Kriegsministerium zurück ${ }^{15}$. Aristide Briand, der dritte Regierungschef der Jahre 1924-26, der jetzt dem Kabinett der Union nationale angehörte, behielt sein Außenministerium 16. Mit Georges Leygues (Républicains de Gauche), der Marineminister wurde, erreichte das Kabinett die in der Geschichte der Dritten Republik einzigartige Zahl von - einschließlich Poincaré - sechs ehemaligen Ministerpräsidenten als Ressortchefs. Louis Marin, parlamentarischer Führer der konservativen Union républicaine démocratique, wurde Pensionsminister, der eben erwähnte Albert Sarraut Innenminister. Bezeichnend für den „überparteilichen“ bzw. auch „überfraktionellen" Zuschnitt des Kabinetts, aber auch für die Chancen eines individuell agierenden Abgeordneten ist die Berufung André Tardieus in das Ministerium

12 Vgl. zu dem Vorgang Botschaftsbericht-Paris (Hoesch), Tel. Nr. 736, 23. 7. 1926, S. 2; PA AA Berlin, R 70717; Bonnet, Vingt ans, S. 122; Herriot, Jadis 2, S. 253, erwähnt die Konsultation mit seiner Partei nicht.

13 Ähnlich in dramatischen Worten auch Herriot, Jadis 2, S. 253.

14 So auch Note Jean, 24. 7. 1926; AN Paris, F7 12954, Bl. 908.

15 Über die unmittelbaren Reaktionen in der Fraktion und Partei der Républicains-socialistes ist nichts bekannt. Allgemein zum Unverständnis in der Partei vgl. Billard, Parti républicain-socialiste, S. 412.

16 Nach Hoesch war die Aufforderung an Briand, das Außenministerium zu übernehmen, ,wie Berthelot mir sagte, eine der ersten Handlungen Poincarés“. Briand erhielt offenbar die Zusicherung, daß keine außenpolitische Kursänderung vorgesehen sei. Botschaftsbericht-Paris (Hoesch), Tel. Nr. 736, 23. 7. 1926, S. 2; PA AA Berlin, R 70717. 
für öffentliche Arbeiten ${ }^{17}$. Ähnlich wie bei Herriot diente auch diese Personalentscheidung vor allem der Ausschaltung eines möglichen parlamentarischen Oppositionsführers ${ }^{18}$. Beide - Tardieu und Herriot - hatten, daran sei erinnert, bereits Anfang 1922, bei der ersten Rückkehr Poincarés in das Amt des Ministerpräsidenten, Angebote zum Kabinettseintritt erhalten, und damals abgelehnt ${ }^{19}$. Die weiteren Ressorts wurden besetzt von Maurice Bokanowski (Gauche républicaine démocratique, Handel), Henri Queuille (Parti radical, Landwirtschaft), Léon Perriot (Senator der Gauche démocratique, Kolonien) und André Fallières (Gauche radicale, Arbeit). Poincaré selbst übernahm neben der Ministerpräsidentschaft das Finanzministerium, das Schlüsselressort für die Lösung der Währungskrise ${ }^{20}$.

Insgesamt ergab diese Kabinettsbildung ein sehr ausgeglichenes politisches Profil: Zwei von 13 Ministern - erneut hatte Poincaré wie schon im März 1924 in haushälterischer Symbolik nur ein relativ kleines Kabinett gebildet ${ }^{21}$ - gehörten der radikalen Kammerfraktion an, zwei den Républicains-socialistes, je einer den Fraktionen der Gauche radicale, der Républicains de Gauche, der Gauche républicaine démocratique und der Union républicaine démocratique. Alle bürgerlichen Kammerfraktionen mit Ausnahme der beiden kleinsten Gruppen der Gauche indépendante und der Démocrates waren damit in der Regierung vertreten. Hinzu kamen der fraktionslose Kammerabgeordnete Tardieu sowie je zwei Minister aus den Senatsfraktionen der Gauche démocratique und der Union républicaine. Glaubt man den Erinnerungen Herriots, dann hatte Poincaré von vornherein einen bestimmten fraktionellen Verteilungsplan im Kopf gehabt ${ }^{22}$. Von einer völlig von den Fraktionen losgelösten Regierungsbildung konnte auch im Zeichen der betont überparteilichen Union nationale keine Rede sein. Allerdings, dies sei ausdrücklich betont, war Poincarés Kabinett keine Koalition der bürgerlichen Fraktionen, sondern eine Zusammenstellung renommierter Persönlichkeiten unterschiedlicher politischer Couleur.

$\mathrm{Da}$ dem Kabinett insgesamt vier Radicaux ${ }^{23}$ und zwei Républicains-socialistes angehörten, lag das politische Schwergewicht deutlich weiter links als in allen Re-

17 Nach Roth, Poincaré, S. 486, verlor der „clemenciste“ Tardieu mit der Annahme des Amtes auch das Wohlwollen seines Mentors Georges Clemenceau.

18 Vgl. ebd., S. 4f.: „Poincaré hat ihn, trotzdem er in Kammer keine feste Anhängerschaft hat, deshalb herangezogen, weil er in ihm den Mann sah, der vielleicht geneigt und fähig gewesen wäre, gegen Kabinett Poincaré in schwierigen Momenten eine Opposition in der Mitte und Rechts zusammenzuschweißen." Tardieu war zum damaligen Zeitpunkt fraktionslos. Vgl. ebd., PA AA Berlin, R 70717, Bl. 198f. - Bonnefous, Histoire 4, S. 166, bezeichnet ihn fälschlicherweise noch als Républicain de Gauche. Ohne nähere Angaben bleibt Junot, André Tardieu.

$19 \mathrm{Vgl}$. oben S. $357 \mathrm{f}$. Herriot hatte damals wohl auf Druck der Partei abgelehnt, obgleich der Bruch mit dem Bloc national noch nicht vollzogen war.

20 Dazu kamen noch die finanzpolitischen Kompetenzen aus dem aufgelösten Ministerium für befreite Gebiete.

21 Insbesondere wurden alle Staatssekretärsposten gestrichen.

22 Herriot, Jadis 2, S. 253, zu dem Gespräch mit Poincaré am 23. 7. 1926: „Il m'énonçait son intention de constituer non un cabinet politique mais un cabinet national, un ministère du franc. Il y réservait quatre départements au parti radical et radical-socialiste, deux au parti socialiste indépendant [Républicains-socialistes], un à la gauche radicale."

23 Neben den beiden radikalen Kammerabgeordneten auch die beiden Senatoren der Gauche démocratique. 
gierungen des Bloc national24, was hinter den Kulissen für einige Verstimmung auf der parlamentarischen Rechten sorgte ${ }^{25}$. Poincaré, der sich 1922-24 immer gegen eine zu strikte Definition seines parlamentarischen Regierungslagers im Sinne eines auf das Mitte-rechts-Spektrum verengten Bloc national gewehrt hatte, war durch diesen Ausbau des linken Kabinettsflügels seinem Ideal einer überparteilichen Regierung sehr nahe gekommen.

Auch wenn die breite und prominent besetzte Ministerliste einen persönlichen „Triumph" für den vor zwei Jahren abgewählten Poincaré bedeutete und ihn, wie der deutsche Botschafter formulierte, "zum präsumptiven Retter Frankreichs“ machte ${ }^{26}$, die parlamentarische Stimmungslage blieb zunächst noch skeptisch. $\mathrm{Zu}$ stark war die Erschütterung über die zurückliegende Talfahrt des Franc und die akuten Haushaltsprobleme, zu unsicher erschienen auch die Aussichten der Regierung Poincaré. Gegnerische Stimmen unter den Abgeordneten prophezeiten bereits ernsthafte Probleme nach der parlamentarischen „rentrée“ im Herbst, und mancher spekulierte bereits über eine kommende Regierung Caillaux ${ }^{27}$. Ein Bericht der Notes Jean konstatierte am 24. Juli verbreiteten Pessimismus "dans les milieux parlementaires“28: „On n'y parle plus que d'organiser le salut, comme si nous étions à la veille d'une catastrophe." Morinaud habe es daher leicht gehabt, seine „intergroupe“ zu rekrutieren. Der allgemeine Tenor sei: „[...] la situation est tragique“. Eine nachhaltige Schockwirkung hatten offenbar die Demonstrationen vor dem Palais-Bourbon am 21. Juli entfaltet. Die Abgeordneten sorgten sich, so der eben zitierte Bericht, insbesondere auch um die Haltung der öffentlichen Meinung und fürchteten, zumindest in Paris, antiparlamentarische "mouvements" 29 .

Am 27. Juli präsentierte sich die neue Regierung vor der Abgeordnetenkammer, die mit Raoul Péret (Gauche radicale) inzwischen wieder ihren Präsidenten aus den Zeiten des Bloc national hatte ${ }^{30}$. In einer kurzen Regierungserklärung betonte der neue Président $d u$ conseil ausdrücklich den „esprit de réconciliation nationale", in dem das Kabinett angesichts der nationalen Notlage gebildet worden sei $^{31}$. In bezeichnender Personalisierung sprach er dabei nicht vom Zusammenwirken unterschiedlicher Parteien, sondern von „Männern, die sich vereint hätten, um im Gleichklang für das Werk des öffentlichen Wohls zu arbeiten“, und deren frühere Meinungsverschiedenheiten jetzt irrelevant seien ${ }^{32}$. Sehr allgemein

24 Vgl. auch Übersicht im Anhang, Tab. 6.

25 Note Jean, 24. 7. 1926; AN Paris, F7 12954, Bl. 908: „A droite, on trouve généralement que $M$. Poincaré, dans son ardent désir bien connu de rentrer en grâce auprès des gauches, a fait à celles-ci la part un peu large." Unwillen erregte v.a. dic Aufnahme von Herriot.

26 Botschaftsbericht-Paris (Hoesch), Tel. Nr. 736, 23. 7. 1926, S. 6; PA AA Berlin, R 70717.

27 Note Jean, 24. 7. 1926; AN Paris, F7 12954, Bl. 906. Die Hoffnungen wurden wohl von Caillaux selbst genährt; vgl. Note Jean, 28. 7. 1926, ebd., Bl. 922.

28 Note Jean, 24. 7. 1926; Bl. 908.

29 Ebd.: „Les députés, en effet, commencent à se demander si le public, si le peuple ne va pas s'en mêler et s'ils ne vont pas voir, à Paris tout au moins, des ,mouvements' contre eux."

30 Zur Wahl am 22.7. 1926 vgl. JO, Débats, Chambre 1926, S. 3033 f. Péret siegte im zweiten Wahlgang mit 227 zu 215 gegen den Sozialisten Bouisson.

31 Ebd., S. 3036.

32 "Chacun des hommes qui se sont rapprocher pour travailler, d'un même cœur, à cette œuvre de salut public, a considéré qu'il avait le devoir d'y consacrer, en ce moment, toute sa pensée et toutes 
umriß Poincaré die Grundzüge des vorgelegten Finanzprojekts, mit dem das Budget für 1926 und 1927 gesichert werden sollte und das im wesentlichen auf eine deutliche Erhöhung indirekter Steuern und auf energische Sparmaßnahmen zielte $^{33}$. Die Bewältigung der „dette flottante“ kurzfristiger Anleiherückzahlungen sollte, wie dies auch schon Caillaux geplant hatte, einer autonomen steuerfinanzierten "Caisse d'amortissement" übertragen werden. Im wesentlichen blieb die neue Regierung damit in Bahnen, die schon durch diverse Finanzvorlagen der Regierungen Painlevé und Briand mit wenig Erfolg angelegt worden waren ${ }^{34}$. Das heikle Thema einer formellen Abwertung des Franc sparte Poincaré geschickt aus.

$\mathrm{Zu}$ grundsätzlichen parlamentarischen Reaktionen auf die Regierungserklärung kam es kaum, denn Poincaré hatte - gewissermaßen als Zeichen tatkräftiger Entschlossenheit und in Wiederaufnahme seiner Praktiken von 1923/2435 - sofort die Vertagung der anstehenden Interpellationen beantragt und dies mit der Vertrauensfrage verbunden. Die vorhergehende Geschäftsordnungsdebatte wurde allerdings von dem sozialistischen Abgeordneten Adéodat Compère-Morel zu bitteren Vorwürfen gegen die einstigen Partner im Linkskartell sowie zu einer scharfen Absage an die Regierung der Union nationale genutzt: „Nous savons trop par expérience que, sous le couvert de la politique dite union nationale ou républicaine, c'est la pire des politiques anti-démocratiques et anti-ouvrières que vous allez mener. Devant cette politique, le parti socialiste ne cessera de se dresser pour dénoncer à la petite bourgeoisie, au monde du travail manuel et intellectuel qui en fera le frais. “36 Konkret richtete sich die Kritik vor allem gegen die geplante Erhöhung indirekter Steuern. Sehr schnell hatte sich die SFIO-Fraktion, die hier in unmittelbarer Konkurrenz zu den wie stets aktiven Kommunisten stand ${ }^{37}$, damit wieder in jene klare Oppositionsrolle zurückbegeben, die schon während der Jahre des Bloc national für eine gleichermaßen plakative wie systemloyale Ausfüllung der Alternativfunktion gesorgt hatte ${ }^{38}$.

Das anschließende Vertrauensvotum erbrachte, wie zu erwarten war, mit $358 \mathrm{zu}$ 131 Stimmen bei 64 Enthaltungen eine erheblich breitere Mehrheit als seit Mai 1924 üblich:

ses forces. Il pourra se poser plus tard d'autres questions sur lesquelles ces hommes différeraient d'opinion [...]. “ Ebd.

33 „Loi ayant pour objet la réalisation d'économies, la création de nouvelles ressources fiscales et diverses mesures d'ordre financier."

34 Vgl. hierzu auch Note Jean, 28. 7. 1926; AN Paris, F7 12954, Bl. 923: „On fait remarquer que le plan financier de M. POINCARE ne comporte aucune indication émanent de son initiative ni aucune forme nouvelle de méthode./ On fait surtout ressortir dans les milieux radicaux et socialistes que ce plan avait été presque entièrement élaboré par M.M. PAINLEVE, LOUCHEUR et DOUMER."

35 Vgl. Kap. I.2 (Zweiter Teil, B).

36 JO, Débats, Chambre 1926, S. 3038 f., Zitat S. 3038.

37 Letztere warfen dem ehemaligen Kartell Verrat vor. So Renaud Jean am 27. 7. 1926 : „Le pays vous a confié son pouvoir, mais il vous l'a confié pour accomplir une tâche qui'il vous avait tracée. Vous n'avez pas le droit d'extorquer ce pouvoir pour réaliser la tâche contrairc." Ebd., S. 3037.

38 Vgl. zur Annahme dieser Rolle auch LP, 6. 8. 1926, S. 1, „Les Socialistes contre le Bloc National“. In der Literatur wird dies verkannt. 
Tab. 22: Votum der Abgeordnetenkammer am 27. 7. 1926: Investitur Regierung Poincaré $I V^{39}$

\begin{tabular}{lrrrrrr}
\hline Fraktion & Abg. & ja & nein & $\begin{array}{l}\text { keine } \\
\text { Teil- } \\
\text { nahme }\end{array}$ & beurl. & $\begin{array}{l}\text { Kohä- } \\
\text { renz }\end{array}$ \\
\hline Communistes & 28 & - & 28 & - & - & $100 \%$ \\
Socialistes (SFIO) & 97 & - & 96 & - & 1 & $100 \%$ \\
Républicains-socialistes & 40 & 22 & 1 & 14 & 3 & $59 \%$ \\
Radicaux et radicaux-socialistes & 136 & 76 & 2 & 49 & 9 & $60 \%$ \\
Gauche radicale & 40 & 38 & - & 1 & 1 & $97 \%$ \\
Démocrates & 14 & 14 & - & - & - & $100 \%$ \\
Gauche indépendante & 16 & 16 & - & - & - & $100 \%$ \\
Gauche républicaine démocratique & 34 & 34 & - & - & - & $100 \%$ \\
Républicains de Gauche & 32 & 31 & - & - & 1 & $100 \%$ \\
Union républicaine démocratique & 102 & 102 & - & - & - & $100 \%$ \\
"Aucun groupe“ & 27 & 23 & 3 & - & 1 & - \\
"Non inscrits“ & 4 & 2 & 1 & - & 1 & - \\
\hline Gesamt & 570 & 358 & 131 & 64 & 17 & - \\
\hline
\end{tabular}

Während das Mitte-rechts-Spektrum sowie die Gauche radicale nahezu geschlossen für das neue Kabinett votierten, reichte die Zahl der Nein-Stimmen kaum über die Opposition der Sozialisten und Kommunisten hinaus. Radicaux und Républicains-socialistes zeigten sich allerdings trotz des Regierungseintritts führender Parteivertreter gespalten: Jeweils etwa $60 \%$ der anwesenden Abgeordneten sprachen der Regierung ihr Vertrauen aus, etwa $40 \%$ enthielten sich bzw. votierten in Einzelfällen mit Nein. Beide Fraktionen befanden sich damit weiterhin in einem Zustand der Zerrissenheit, wie er auch schon während der zurückliegenden Monate geherrscht hatte. Glaubt man einem polizeilichen Informantenbericht, dann war zunächst sogar eine noch deutlichere Opposition der Radicaux zu erwarten gewesen. Etwa die Hälfte der Fraktion wolle, so wurde am 24. Juli prophezeit, gegen die Regierung und damit auch gegen ihren eigenen Parteivorsitzenden stimmen. Am Verhalten Herriots werde viel Kritik geübt, sein Regierungseintritt gelte als ein "Sich-aus-der-Verantwortung-Stehlen“ („dérobade“), viele Abgeordnete sprächen hinsichtlich der Parteiführung bereits von einem möglichen Nachfolger Caillaux ${ }^{40}$. In ähnliche Verwirrungen wurde offenbar auch die Fraktion der Républicains-socialistes gestürzt ${ }^{41}$.

Nach dem Vertrauensvotum beschloß die Kammer mit sehr breiter Mehrheit $418 \mathrm{zu} 31$ bei 104 Enthaltungen -, für das anstehende finanzpolitische Gesetzgebungsverfahren die erst vor wenigen Tagen eingeführte „procédure d'extrême

39 Liste der namentlichen Abstimmung in JO, Débats, Chambre 1926, S. 3056 f.; Aufstellung nach Fraktionen aus LT, 29. 7. 1926, S. 2, "Le scrutin sur la confiance“.

40 Note Jean, 24. 7. 1926; AN Paris, F7 12954, Bl. 906. Kritisiert wurde insbesondere auch Herriots Brief an Cazals. Zur angeblich bestehenden "haine farouche" zwischen Herriot und Caillaux vgl. Note Jean, 27. 7. 1926; ebd., Bl. 920.

41 Das geteilte Votum läßt sich als Beginn eines Auflösungsprozesses deuten, der schließlich 1928 zur neuerlichen Parteispaltung führte. Vgl. Billard, Parti républicain-socialiste, S. 414-427. 
urgence" anzuwenden ${ }^{42}$. Die SFIO-Fraktion übte hierbei Stimmenthaltung. $\mathrm{Ob}$ dies tatsächlich ein verkappter Vertrauensbeweis für die neue Regierung war ${ }^{43}$, erscheint fragwürdig. Möglicherweise wollten die Sozialisten nicht gegen die Anwendung einer Verfahrensänderung stimmen, die sie - als Alternative zur Ermächtigungspolitik - selbst initiiert hatten ${ }^{44}$.

Auch wenn der erste Auftritt des neuen Kabinetts in der Abgeordnetenkammer durch die breite Stimmenthaltung bei den Radicaux etwas getrübt worden war ${ }^{45}$, insgesamt hatte es seine Präsentation erfolgreich bestanden. Ein Polizeibericht aus dem Palais Bourbon vermeldete denn auch, entgegen der bei der Regierungsbildung noch vorherrschenden Skepsis, eine „bonne impression dans les milieux politiques et financiers" 46 . Klar für die Regierung sprach zudem die überraschend deutliche Erholung des Franc: Der Kurs des Dollars war bis zum 28. Juli wieder auf einen Wert von rund 42 Francs gesunken ${ }^{47}$.

\section{Beschränktes Ermächtigungsgesetz und andere Demonstrationen der Kooperation zwischen Regierung und Abgeordnetenkammer}

Die am 27. Juli eingebrachte Finanzvorlage, deren finanzpolitischer Ansatz bereits umrissen wurde, ist für unsere Thematik vor allem in einem Punkt von Interesse. Die 1924 von Poincaré gegen heftige Widerstände durchgesetzte, in der Praxis allerdings nie genutzte parlamentarische Ermächtigung fand nun eine Nachfolgeregelung, die das Mittel der décrets-lois erstmals in den „Normalbetrieb“ der Dritten Republik einführte. Besondere Beachtung verdient dabei der Umstand, daß die neuen Ermächtigungen parlamentarisch und in der politischen Öffentlichkeit so gut wie überhaupt nicht mehr diskutiert wurden. Abgesehen von älteren juristischen Spezialuntersuchungen sind sie im übrigen auch in der Literatur kaum wahrgenommen worden ${ }^{48}$.

Während und auch unmittelbar nach der Regierungsbildung Poincarés war in parlamentarischen Kreisen und in der politischen Öffentlichkeit von décrets-lois zunächst überhaupt keine Rede gewesen. Vermutlich bemühte sich der neue Ministerpräsident sofort, möglichen Diskussionen die Grundlage zu entziehen und sich in dieser Beziehung von der gescheiterten Regierung Briand-Caillaux abzusetzen. So wußte ein Polizeibericht vom 24. Juli, daß im Palais Bourbon die Auffassung herrschte, Poincaré werde keine pleins pouvoirs beantragen ${ }^{49}$. Auch bei

42 JO, Débats, Chambre 1926, S. 3042 f., Liste der namentlichen Abstimmung ebd., S. $3057 \mathrm{f}$.

43 Bonnefous, Histoire 4, S. 169. Ähnlich im Anschluß daran Mayeur, Vie politique, S. 185, der daraus ableitet, die Opposition der Sozialisten sei „courtoise et sans passion“ geblieben.

44 Vgl. oben S. 487.

45 Vgl. etwa den besorgten Kommentar in LT, 29. 7. 1926, S. 1, „La séance d'hier“.

46 Note Jean, 28. 7. 1926; AN Paris, F7 12954, Bl. 923.

47 Vgl. LT, 29. 7. 1926, S. 5, "Côte des changes“ (Börsennotierung). Allgemein zur Erholung vgl. Bonnefous, Histoirc 4, S. 167.

48 Maßgeblich ist sicher auch die Nichterwähnung bei Bonnefous, Histoire 4, S. 169-171, wo nur allgemein die Durchsetzung des Finanzprojekts dargestellt wird. Auch in Berstein, Histoire 2, sowie in ders., Herriot, wird das Thema völlig übergangen. Vgl. zur Ermächtigung v.a. Mignon, La pratique des décrets-lois; Rusu, Les décrets-lois, S. 154-156; Hippel, Entwicklung, S. 192 f.; Rothenfluh, Notrecht, S. $71 \mathrm{f}$.

49 Note Jean, 24. 7. 1926; AN Paris, F7 12954, Bl. 907: „[...] on prétend qu'il [Poincaré] ne réclamerait pas les pleins pouvoirs pour appliquer ses mesures financières". 
der parlamentarischen Präsentation der neuen Regierung in der Abgeordnetenkammer blieb das Thema im Plenum unerwähnt. Der Regierungschef wies statt dessen lediglich allgemein auf die notwendige "collaboration immédiate et résolue entre le Gouvernement et les Chambres" hin, was wie eine Absage an die von Caillaux geforderten pleins pouvoirs gedeutet werden konnte ${ }^{50}$. Der neue Kammerpräsident Péret hatte zuvor die Notwendigkeit betont, das parlamentarische System gegen Kritik zu verteidigen und in diesem Zusammenhang ausdrücklich die beschlossene Verfahrensbeschleunigung gelobt ${ }^{51}$.

Zur erwähnten Finanzvorlage der Regierung gehörten jedoch auch eine ganze Reihe inhaltlich genau festgelegter parlamentarischer Ermächtigungen. Am wichtigsten war Artikel 1, der unbegrenzte Möglichkeiten von Verwaltungseinsparungen vorsah: „Le gouvernement est autorisé à procéder par décrets, jusqu'au 31 décembre 1926, à toutes suppressions ou fusions d'emplois, d'établissements ou de services. Lorsque ces mesures nécessiteront soit des modifications à des organisations, formalités ou procédures fixées par la loi, soit des annulations ou transferts de crédits, elles ne pourront être rapportées que par un texte législatif." ${ }^{2}$

In ihrem materiellen Kern ähnelte diese Ermächtigung dem heiß umstrittenen Gesetz vom 22. März 1924 („toutes les réformes et simplifications administratives que comportera la réalisation de ces économies") ${ }^{53}$. Weitere spezielle Ermächtigungen in den Artikeln 2 und 3 des Finanzprojekts verliehen der Regierung Freiheiten bei der Anpassung von Zöllen und staatlichen Tarifen an die inflationäre Entwicklung54.

Infolge des angewandten Verfahrens der „extrême urgence“ kam die Vorlage sofort in die Finanzkommission der Abgeordnetenkammer. Im Mittelpunkt der dortigen Beratungen stand am 28. Juli eine ausführliche Anhörung Poincarés55. Während die geplanten finanzpolitischen Maßnahmen, insbesondere die Erhöhung indirekter Steuern, heftige, vor allem von den Sozialisten vorgebrachte Kritik fanden, spielte das grundsätzliche verfassungsrechtliche Problem der Ermächtigungen hier so gut wie keine Rolle. Äußerst geschickt vermittelte der Regierungschef den Eindruck, die legislative Funktion des Parlaments so weit wie möglich wahren zu wollen. In gewisser Weise distanzierte er sich dabei sogar von dem etwas pauschaler formulierten - Ermächtigungsgesetz vom 22. März 1924: „Je n’ai

50 JO, Débats, Chambre 1926, S. 3036. Poincaré spieltc hier offenbar auf Herriot an, der am 17.7. 1926 in seinem furiosen Auftritt vor der Kammer ausdrücklich die Forderung nach „collaboration" gestellt hatte.

51 Ebd., S. 3035: „Il faut aussi que par une action soutenue, nous défendions ensemble l'institution parlementaire contre certaines critiques (Interruptions ...); la discipline et la méthode, que faciliteront d'ailleurs les modifications apportées hier par la Chambre à son règlement, apparaissent plus que jamais nécessaires."

32 Zitiert nach LT, 29. 7. 1926, S. 3, „Le projet financier du gouvernement“. In JO, Débats, Chambre 1926, Annexes, fehlt der Gesetzentwurf. Bis auf den Schluß - vgl. unten S. 509 zur Einfügung einer Klausel, dic eine parlamentarische Ratifizierung vorsieht - identisch mit dem späteren Text. Vgl. Anhang, 7.2.

53 Vgl. Text im Anhang, 7.2.

54 Nach LT, 29.7. 1926, S. 3, „Le projet financicr du gouvernement“.

55 Stenographisches Protokoll in AAN Paris, Procès-verbaux de la Commission des Finances, XIII Législature, 28. 7. 1926. Vgl. auch ausführliche Berichte in LT, 30. 7. 1926, S. 3, "La Chambre“, S. 6, "Dépêches particulières de la Chambre“. Zusammenfassender Bericht der Kommission für die Kammer abgedruckt in LT 32. 7. 1926, S. 3f., "Le projet financier du Gouvernement“. 
pas repris le texte que les Chambres avaient voté sous le nom fâcheux que je n'avais jamais accepté de décrets lois. Je n'ai pas voulu remettre la Chambre actuelle en présence d'un texte que beaucoup de députés avaient alors abandonné. "56

Eine in der Vorlage versteckte Einschränkung der parlamentarischen Kontrollfunktion wurde in der Ausschußberatung wieder rückgängig gemacht. Louis de Chappedelaine (Gauche radicale), Rapporteur général der Finanzkommission, hatte beanstandet, daß im Entwurf die Klausel einer notwendigen parlamentarischen Ratifizierung der erlassenen Verordnungen fehlte $\mathrm{e}^{57}$. Der zunächst vorgesehene Hinweis, getroffene legislative Maßnahmen könnten nur mittels eines Gesetzes wieder aufgehoben werden, wurde nun wie bei der Ermächtigung von 1924 durch eine Ratifizierungsformel ersetzt ${ }^{58}$.

Das Finanzprojekt mit seinen Ermächtigungen passierte den Ausschuß bis zum 30. Juli unter kleinen Veränderungen weitgehend problemlos und wurde zuletzt mit 19 zu 13 Stimmen bei einer Enthaltung gebilligt. Die Gegenstimmen kamen von Sozialisten und Kommunisten sowie von der überwiegenden Zahl der anwesenden Radicaux und Républicains-socialistes. Konservative Kreise sahen hierin in gewisser Vorahnung der späteren Volksfront - bereits ein Indiz für ein drohendes „Neo-Kartell“ unter kommunistischer Beteiligung59.

Bevor das Finanzprojekt in die Plenarberatung kam, beschloß die Abgeordnetenkammer am 30. Juli auf Antrag des Geschäftsordnungsausschusses mit $298 \mathrm{zu}$ 180 Stimmen eine weitere Verfahrensänderung, die in die Tradition des französischen Parlamentarismus eingriff60. Für die Plenarbehandlung der Finanzvorlage und der darin enthaltenen Ermächtigung wurde das Gesetzgebungsverfahren nun nochmals beschleunigt ${ }^{61}$. Ausnahmsweise sollte es - entgegen der gültigen Geschäftsordnung - während der parlamentarischen Beratung der Finanzvorlage einzelnen Abgeordneten nicht möglich sein, ein amendement einzubringen. Auf

56 Stenographisches Protokoll; AAN Paris, Procès-verbaux de la Commission des Finances, XIIIe Législature, 28. 7. 1926, S. 52f. der "audition“.

57 Ebd., S. 8, des „examen“ der Kommission.

58 „Lorsque ces mesures nécessiteront soit des modifications à des organisations, formalités ou procédures fixées par la loi, soit des annulations ou transferts de crédits, elles devront être soumises à la ratification des Chambres dans un délai de trois mois." Auch die Ermächtigungen der dreißiger Jahre besaßen dann ähnliche Regelungen.

59 Vgl. Bericht und Kommentar in LT, 31. 7. 1926, S. 1. - Die Gegenstimmen verteilten sich im einzelnen: Sozialisten 5 (von 5 Anwesenden), Kommunisten 2 (von 2), Radicaux (5 von 7), Républicains-socialistes 1 (von 3 bei einer Enthaltung).

60 Der Kern dieser Regelung lautete: „Sur le projet de rétablissement financier déposé par le Gouvernement à la séance du 27 juillet 1926, aucun amendement ne sera recevable en séance publique. Seule la commission des finances pourra soumettre à la Chambre, en vue de la discussion en séance publique, des modifications au projet. “ Debatte in JO, Débats, Chambre 1926, S. 3062-3067. Liste der namentlichen Abstimmung ebd., S. $3076 \mathrm{f}$.

68 In einer etwas heiklen Situation waren die Sozialisten, denn auch diese Regelung ging letztlich zurück auf den bereits mehrfach erwähnten Vorstoß Renaudels zur Beschleunigung des legislativen Verfahrens. Allerdings wies dessen Vorschlag, der jetzt als „contre-projet“ (Text ebd., S. 3066) wieder in die Beratung eingebracht wurde, zwei markante Unterschiede auf. Zum einen sollte hier keine Ausnahmeregelung geschaffen werden, sondern eine dauerhafte Reform der Geschäftsordnung. Zum anderen wäre der Eingriff in die Tradition des Gesetzgebungsverfahrens weit geringer gewesen. Jedes amendement sollte demnach zunächst an den zuständigen Ausschuß geleitet werde, der es dann - gleichsam als Filter gegen chancenlose Anträge - mit Zweidrittelmehrheit zurückweisen konnte. Die Plenardiskussion wäre so auf eine Auswahl erfolgversprechender Änderungsanträge beschränkt worden. 
diese Weise konnten zeitraubende und in den letzten Jahren oftmals zur Blockade eingesetzte Diskussionen um Änderungsanträge vermieden werden. Gleichzeitig war aber auch eine wesentliche Antriebskraft der klassischen Deliberation suspendiert. Statt dessen sollten Änderungsvorschläge nur durch die Finanzkommission vorgelegt werden. Da hier Mehrheitsentscheidungen notwendig waren, blieb die legislative Mitwirkung somit de facto auf die neue Kammermehrheit beschränkt. Während sich die Kommunisten, die das destruktive Potential von amendements bestens beherrschten, in der diesbezüglichen Kammerdebatte als Verteidiger der parlamentarischen Tradition aufspielten, wurde die Ausnahmeregelung von ihren Initiatoren als Ausdruck patriotischer Disziplin gepriesen ${ }^{62}$.

Am 31. Juli wurde das Finanzgesetz im Eilgang durch die Abgeordnetenkammer getrieben ${ }^{63}$. Eine grundsätzliche Diskussion über die Ermächtigung war nicht einmal mehr ansatzweise zu erkennen, zumal es Poincaré bei Begründung des Artikels 1 geschickt verstand, den Begriff „décret" zu vermeiden ${ }^{64}$. Im Rahmen der allgemeinen finanzpolitischen Diskussion brachte Auriol für die Minderheit des Finanzausschusses allerdings eine relativ ausführliche und durchaus scharfe Kritik am Konzept der Regierung vor ${ }^{65}$. Erneut war dies eine Gelegenheit, die Oppositionsrolle der Sozialisten zu demonstrieren und eine inhaltliche Alternative zur Regierung der Union nationale zu verdeutlichen. Das Gesamtprojekt wurde schließlich mit 295 zu 188 Stimmen bei 49 Enthaltungen gebilligt ${ }^{66}$. Die Masse der Gegenstimmen stammte, ebenso wie einen Tag zuvor beim Votum über die Suspendierung des Rechts auf amendements, von Kommunisten, Sozialisten und einer Minderheit der tief gespaltenen Radicaux und Républicains-socialistes ${ }^{67}$.

Die Behandlung im Senat war angesichts der in der Gauche démocratique herrschenden Sympathien für die neue Regierung nur noch eine Formsache. Auch hier passierte die Vorlage zunächst die Finanzkommission, um dann am 3. August im Plenum mit 245 zu 8 Stimmen in der von der Kammer verabschiedeten Form ohne jede Änderung gebilligt zu werden ${ }^{68}$. Noch am gleichen Tag trat das Finanzgesetz mit seinen Ermächtigungen in Kraft.

Das Instrument einer legislativen parlamentarischen Ermächtigung wurde nun - abgesehen von den speziellen Sonderfällen während des Weltkrieges und im Zuge Integration von Elsaß-Lothringen - erstmals in der Geschichte der Dritten Republik zum Erlaß von décrets-lois eingesetzt. Bei aller Bedeutung als Tabu-

62 Vgl. va. die Äußerungen von Jean Garchery (Groupe communiste) und von Léon Baréty (Républicains des gauche), Präsident der Geschäftsordnungskommission. Ebd., $3063 \mathrm{f}$.

63 JO, Débats, Chambre 1926, S. 3080-3092 (erste Sitzung), S. 3094-3115 (zweite Sitzung).

64 En passant bemerkte Poincaré zum Thema „Einsparungen“: „L'article 1er du projet, qu'a bien voulu adopter la commission des finances dans un texte légèrement modifié mais que $j$ 'accepte sans difficulté, nous en donnera les moyens. “ Ebd., S. 3088.

65 Ebd., S. 3083-3086. Vgl. auch LP, 6. 8. 1926, S. 1, "Les Socialistes contre le Bloc national“.

66 Liste der namentlichen Abstimmung in JO, Débats, Chambre 1926, S. $3122 \mathrm{f}$.

67 Vgl. das nach Fraktionen differenzierte Ergebnis in LT, 2. 8. 1926, S. 2, "Le scrutin sur l'ensemble du projet financier". Demnach stimmten 31 Radicaux gegen das Projekt - darunter als prominentester Name Daladier -, 32 enthielten sich, 17 waren beurlaubt, und 56 votierten für die Regierung. Bei den Républicains-socialistes gab es 12 Nein-Stimmen, eine Enthaltung, 8 Beurlaubungen und $19 \mathrm{Ja}$-Voten.

68 Vgl. den zusammenfassenden Bericht in LT, 5. 8. 1926, S. 3, „Le projet financier devant le Sénat“. Unter den Gegenstimmen waren auch die fünf in der zweiten Kammer vertretenen Sozialisten. 
bruch, der dann in den dreißiger Jahren weitreichende Folgen für die legislative Funktion des Parlaments haben sollte, ist doch auch der relativ enge Charakter der Ermächtigung bemerkenswert. Statt eines Blankoschecks im Sinne von pleins pouvoirs blieben die Ziele der Ermächtigung genau umrissen. Von „pouvoirs dictatoriaux " 69 kann allenfalls für das Feld einer Verwaltungsreform gesprochen werden. Und selbst in dieser Beziehung war die Vollmacht - Poincaré hat in der Sitzung der Finanzkommission der Kammer mit Recht darauf hingewiesen - etwas präziser als die Vorgängerregelung von 1924. Dank der erwähnten Intervention der Finanzkommission blieb auch die Notwendigkeit der parlamentarischen Ratifizierung erlassener décrets-lois gewahrt.

Obwohl die 1924 noch so heftige verfassungspolitische Diskussion um die $\mathrm{Zu}$ lässigkeit derartiger Ermächtigungen inzwischen weitgehend zum Erliegen gekommen war, zeigte sich hier doch bei allen Beteiligten das Bemühen, die legislative Kompetenz des Parlaments so wenig wie möglich einzuschränken und gleichzeitig seine Kontrollrechte so weit als möglich zu wahren. Ähnlich sensibel wie der klassische französische Parlamentarismus auf Mehrheitsänderungen in der Abgeordnetenkammer reagierte, respektierte der erfahrene Parlamentarier Poincaré mit seiner engen Gesetzesvorlage die parlamentarischen Bedenken, die sich bei der Ermächtigung von 1924 und dann teilweise auch 1926 bei der Ermächtigungsvorlage von Caillaux gezeigt hatten. Letztlich ist hierbei unerheblich, ob dies aus verfassungspolitischer Überzeugung oder aus taktischen Überlegungen geschah. Im Ergebnis trug das Gesetz vom 3. August diesen Bedenken durchaus Rechnung. Eine Gleichsetzung mit dem weiten Ermächtigungsprojekt der Regierung Briand-Caillaux vom 9. Juli, wie sie in Teilen der verfassungsrechtlichen Literatur vorgenommen wird ${ }^{70}$, erscheint daher unzulässig.

Trotzdem bleibt erstaunlich, wie glatt die Ermächtigungsvorlage Poincarés die parlamentarischen Hürden genommen und wie wenig Aufsehen sie in der politischen Öffentlichkeit erregt hat. Bei der 1924 so heftig gegen das Mittel der parlamentarischen Ermächtigung polemisierenden Kammerlinken waren, wie sich schon bei der Beratung der Vorlage von Caillaux gezeigt hatte, die grundsätzlichen Widerstände weitgehend geschwunden. Auch die Camouflage Poincarés, der alles getan hatte, sein Ermächtigungsprojekt als solches unkenntlich zu machen, erzielte wohl Wirkung. Schwer erklärbar ist freilich die Haltung Herriots, der noch zwei Wochen zuvor als einsamer Kämpfer für die traditionellen Rechte des Parlaments aufgetreten war und so zum Sturz der Regierung Briand-Caillaux beigetragen hatte. Als Unterrichtsminister in das Kabinett der Union nationale eingebunden, zeigte der Vorsitzende des Parti radical nicht die geringste Kritik an der neuen Ermächtigung. Vermutlich spielte hier der materielle Unterschied zur weitgefaßten Vorlage des ungeliebten Parteifreundes Caillaux eine wesentliche Rolle. Allerdings ist daran zu erinnern, daß Herriot das der aktuellen Ermächtigung relativ ähnliche Projekt Poincarés von 1924 scharf bekämpft hatte. Das jetzige Schweigen Herriots läßt daher das verfassungspolitische Pathos seines Auftrittes 
vom 17. Juli 1926 etwas fragwürdig erscheinen. Möglich ist freilich auch eine gewisse Resignation angesichts der seither erfolgten politischen Entwicklung ${ }^{71}$.

Eine grundlegende Frage bleibt im Zusammenhang mit der Ermächtigung vom 3. August noch zu beantworten. Warum griff Poincaré, wenn auch in äußerst vorsichtiger Form, erneut zu diesem Mittel, das ihm im Frühjahr 1924 heftige Diskussionen eingebracht und das wenige Wochen zuvor zum Sturz der Regierung Briand geführt hatte? Explizite Begründungen gab der Regierungschef nicht, und zweifellos hätten diese auch seine Taktik behindert, die Angelegenheit so tief als möglich zu hängen. Allerdings fällt es nicht schwer, die Motive zu erschließen. Ähnlich wie 1924 dürfte vor allem eine gewisse Labilität der eigentlich so breiten parlamentarischen Mehrheit eine Rolle gespielt haben. Noch war Poincarés Position in der Abgeordnetenkammer keineswegs so gefestigt wie nach der Stabilisierung des Franc. Die fragile Union nationale mit den Detailproblemen einer landesweit für Unruhe sorgenden Verwaltungsreform zu belasten, wäre ein riskantes Unterfangen gewesen. Hinzu kommt eine symbolische Dimension. Die ungewöhnlich schnell und mit neuartigen Methoden parlamentarischer „Rationalisierung" erreichte Verabschiedung der Finanzvorlage mitsamt ihren Ermächtigungen war auch ein Schauspiel entschlossener politischer Führung und parlamentarischer Disziplinierung, das der Regierungschef gegenüber Finanzmärkten, Öffentlichkeit und Abgeordnetenkammer in Szene setzte.

Diese Feststellung gilt auch für die Inszenierung einer formellen und bislang äußerst seltenen ${ }^{72}$ Verfassungsergänzung, mit der eine autonome Tilgungskasse (Caisse autonome d'amortissement) zur Verwaltung und Rückzahlung der Bons de la défense nationale begründet wurde. Die Idee, zur Bewältigung der kurzfristigen und von den Stimmungslagen der Anleger beeinflußten "dette flottante“ eine von der Regierung unabhängige und über die Tabaksteuer finanzierte Institution zu schaffen, war schon von den Vorgängerregierungen verfolgt worden. Hierfür die Assemblée nationale in Versailles einzuberufen, bedeutete einerseits einen Akt besonderer Vertrauenswerbung, andererseits aber auch ein weiteres Exempel für die Durchsetzungsfähigkeit der neuen Regierung auf verfassungsrechtlich heiklem Terrain.

Nachdem zunächst am 5. und 6. August Abgeordnetenkammer und Senat mit breiten Mehrheiten das Vorhaben einer Caisse autonome gebilligt hatten ${ }^{73}$, folgte einen Tag später die Plenardebatte der Abgeordnetenkammer über die vorgesehene Einberufung der Assemblée nationale ${ }^{74}$. Von Paul Bastid, einem im Prinzip regierungsfreundlichen Abgeordneten der Radicaux, kam dabei ein beschwörender und quer durch die Kammer mit Beifall bedachter Appell an Poincaré, auf die keineswegs zwingende Verfassungsergänzung zu verzichten. Bastid fürchtete, daß es nach einem solchen Präzedenzfall in Versailles zu weiteren verfassungsrecht-

71 Die vorliegende Literatur zu Herriot geht hierauf nicht ein.

72 Seit 1875 gab es nur zwei Fälle: Am 21.6. 1879 wurde der Regierungs- und Parlamentssitz Versailles aufgehoben (Art. 9 des Gesetzes vom 25. 2. 1875); am 14. 8. 1884 wurden diverse Ergänzungen zur weiteren Sicherung der parlamentarischen Republik vorgenommen. Vgl. hierzu Gicquel, Droit constitutionnel, S. $474 \mathrm{f}$. Ansonsten traten Abgeordnetenkammer und Senat nur zur Wahl des Staatspräsidenten als Assemblée nationale zusammen.

73 Kammersitzung am 5.8. in JO, Débats, Chambre 1926, S. 3236-3238.

74 Vgl. Bonnefous, Histoire 4, S. 173-175. 
lichen Vorstößen kommen würde, und nannte an erster Stelle den Versuch eines „démantelement de la forteresse sénatoriale"75. Genau dies zeichnete sich noch in derselben Kammersitzung ab, als Renaudel für die Sozialisten eine Verfassungsergänzung vorschlug, welche die legislative Gleichberechtigung des Senats - „l'un des vices de notre régime parlementaire" - aufgehoben und im Konfliktfall der Abgeordnetenkammer - ähnlich wie heute in der Fünften Republik - die letzte Entscheidung zugewiesen hätte ${ }^{76}$. Angesichts der fatalen Rolle, die der Senat gegenüber dem Linkskartell gespielt hatte, war dies eine sehr naheliegende Initiative. Weder die Bedenken Bastids noch der Vorschlag Renaudels konnten jedoch in der Kammer eine Mehrheit finden 77.

In der Sitzung der Nationalversammlung am 10. August in Versailles scheiterte zunächst der Vorschlag der Sozialisten zur Beschneidung der legislativen Macht des Senats ${ }^{78}$. Die Tilgungskasse erhielt nach grundsätzlicher Aussprache mit 671 zu 144 Stimmen Verfassungsrang (Loi constitutionnelle vom 10. August 1926 in Ergänzung zum Verfassungsgesetz vom 25. Februar 1875). Eine spektakuläre Note gewann die Versammlung durch den Auftritt des Kommunisten (und späteren Faschisten) Jacques Doriot ${ }^{79}$, dem wegen heftiger Polemiken das Wort entzogen wurde und der nach seiner Weigerung, das Rednerpult zu verlassen, zuletzt vom Militärkommandanten der Abgeordnetenkammer nach außen geführt wurde. Nicht nur Poincaré verstand es, die Assemblée nationale effektvoll zu instrumentalisieren.

Wenige Tage später, am 11. August, wurde die parlamentarische Session ordinaire des Jahres 1926 geschlossen. Während der dreimonatigen, bis zum 12 . November dauernden Sommer- und Herbstpause der beiden Kammern setzte die Regierung Poincaré auf dem Verordnungsweg, gestützt auf Artikel 1 des Gesetzes vom 3. August, eine umfangreiche Verwaltungsreform ins Werk, deren Hauptziel es war, für eine Entlastung der Staatsausgaben zu sorgen. Orientiert an den Vorgaben der Commission Louis Marin aus dem Jahre 192380 , erfolgten in mehr als 80 Verordnungen erhebliche Einschnitte in das bestehende Verwaltungsnetz ${ }^{81}$. So wurden zahlreiche Unterpräfekturen, lokale Finanzämter, Gerichte und Gefäng-

75 JO, Débats, Chambre 1926, S. 3245 f., Zitat S. 3245.

76 Ebd., S. 3248-3250, Zitat S. 3248. Konkret sah das sozialistische Projekt eine Ergänzung von Artikel 8 des Verfassungsgesetzes vom 24. 2. 1875 vor. Kernstück war das legislative Primat der Abgeordnetenkammer in einer eventuell nach Änderungen des Senats notwendig werdenden dritten Lesung. Text des Vorschlags ebd., S. 3248. Mit Recht betonte Renaudel, daß damit die französische Verfassung an den Stand anderer Länder angeglichen würde und führte als Vorbild insbesondere die englische, gegen die legislative Macht des Oberhauses gerichtete Verfassungsänderung aus dem Jahre 1911 an. Vgl. ebd., S. 3249.

77 Die von der Regierung beantragte Resolution zum Programm der Nationalversammlung erhielt eine Mehrheit von 395 zu 131 Stimmen (weitgehend nur Sozialisten und Kommunisten), Renaudel erreichte immerhin 166 Stimmen gegen 347. Hier hatte immerhin eine nennenswerte Minderheit der Radicaux für eine Entmachtung des Senats votiert. Liste der namentlichen Abstimmung ebd., S. 3262-3264.

78 Zur Sitzung der Assemblée nationale vgl. ausführlicher Bericht in LT, 12. 8. 1926, S. 3f., „Assemblée nationale"; zusammenfassend Bonnefous, Histoire 4, S. 175-178.

79 Allgemein zu Doriot vgl. Burrin, La dérive fasciste.

80 Vgl. oben S. $391 \mathrm{f}$.

81 Detailliert zu den Verordnungen auf Grundlage des Ermächtigungsgesetzes: Bonnard, Décretslois, S. 11-43; stärker problemorientiert: Bonnefous, Réforme administrative; knapp: Bonnefous, Histoire 4, S. 178-180. 
nisse aufgehoben. Die notwendigen parlamentarischen Ratifizierungen der erlassenen Verordnungen erfolgten im Winter 1926/27 lediglich als rasch vollzogene Formalitäten.

Diese - in den Folgejahren im übrigen wieder weitgehend rückgängig gemachte ${ }^{82}$ - Verwaltungsreform war vor allem eine Demonstration energischen Sparwillens und leistete so neben der tatsächlichen Entlastung des Budgets einen Beitrag zur psychologischen Wiederaufrichtung des französischen Franc. Die vielbeschworene Rückgewinnung des Vertrauens machte seit der Berufung Poincarés zum neuen Regierungschef rasche Fortschritte ${ }^{83}$, ablesbar in einer wieder verstärkten Zeichnung von Staatsanleihen und einer deutlichen Erholung des Währungskurses. Bereits am 4. August, einen Tag nach Inkraftsetzung des Finanzprojektes, war der Preis des Dollars auf rund 35 Francs gefallen ${ }^{84}$, im Dezember lag er schließlich bei rund 25 Francs. Damit war jener Stand erreicht, auf dem sich der Franc im November 1925 vor Eskalation der parlamentarischen Krise befunden hatte.

Tab. 23: Kurs des französischen Franc gegenüber dem Dollar von Juli bis Dezember 1926 im Monatsmittel 85

Monat Dollarpreis in Francs

\begin{tabular}{lr}
\hline Juli & 140,95 \\
August & 35,42 \\
September & 35,05 \\
Oktober & 34,15 \\
November & 29,12 \\
Dezember & 25,33 \\
\hline
\end{tabular}

Die entscheidende Frage war nun, wann und auf welchem Niveau eine formelle Stabilisierung des Franc durchgeführt werden sollte ${ }^{86}$. Poincaré - ursprünglich wie die herrschende finanzpolitische Meinung Anhänger einer "revalorisation“ auf Vorkriegsniveau - akzeptierte nach und nach den schon seit längerem vorsichtig diskutierten und inzwischen auch an der Spitze der Banque de France vertretenen Gedanken einer definitiven Abwertung auf einen ökonomisch sinnvollen Stand. Für die vorliegende Arbeit ist vor allem der Umstand von Interesse, daß dieser Erkenntnisprozeß lange Zeit keinerlei parlamentarischen Niederschlag gefunden hat. Der Regierungschef hielt sich den Kammern und auch der Öffentlichkeit gegenüber mit konkreten Äußerungen über Zeitpunkt und Modalitäten einer

82 Vgl. Bonnefous, Réforme administrative, S. 64-66.

83 Fohlen, Frankreich, S. 107, sieht hierin die Bestätigung, daß der Währungsfall seit 1924 „mehr eine Vertrauensfrage als ein technisches Problem" gewesen sei.

84 LT, 5. 8. 1926, S. 5, "Côte des changes" (Börsennotierung 4. 8. 1926).

85 Zahlen nach Sauvy, Histoire économique 1, S. 445.

86 Zur Stabilisierungsfrage vgl. v.a. Sauvy, Histoire économique 1, S. 83-99; Jeanneney, De Wendel, S. 321-409; Blancheton, Le Pape et l'Empereur, S. 379-450; Mouré, The Gold Standard Illusion, S. 101-144. Die neue These Mourés (vgl. ebd., S. 114), daß Poincaré bewußt auf eine Verzögerung der formellen Stabilisierung gesetzt hat, um die Radicaux weiterhin an sich zu binden, erscheint plausibel, bedürfte aber einer genaueren Überprüfung. 
Abwertung weitestgehend zurück ${ }^{87}$. Umgekehrt fügte sich das Parlament in eine abwartende Rolle. So war das Thema im Dezember 1926 in der Abgeordnetenkammer bei der Beratung und breiten Billigung des Budgets 1927 erstaunlicherweise kaum präsent ${ }^{88}$. Der bisherige Erfolg sprach aus Perspektive des neuen Regierungslagers dafür, auch in dieser Frage auf das Geschick Poincarés zu vertrauen und sich seiner Führung unterzuordnen. Positiv für das Kabinett wirkte zudem der Umstand, daß sich die Haushaltslage, vor allem dank der Erhöhung indirekter Steuern sowie des veränderten Verhaltens auf dem Anleihemarkt, inzwischen in einem relativ günstigen Licht präsentierte.

\section{Die neue Konstellation in der Abgeordnetenkammer}

Seit dem Antritt Poincarés hatte sich auch die parlamentarische Situation weitgehend stabilisiert. Deutlichstes Indiz ist die ungewöhnlich lange, über zwei Jahre bis zum November 1928 dauernde Regierungsdauer des Kabinetts Poincaré IV, die sogar noch etwas über der Amtszeit des Kabinetts Poincaré II (Januar 1922März 1924) lag. Eine breite Mehrheit in der Abgeordnetenkammer sicherte nicht nur die Existenz der Regierung, sondern sorgte - wie sich bei der problemlosen Verabschiedung aller Gesetzesvorhaben zeigte - auch für einen Ausweg aus der legislativen Blockade. Trotz der begrenzten Funktionsabgabe durch die Ermächtigung vom 3. August ist damit auch eine Wiederbelebung der legislativen Funktion des Parlaments zu konstatieren.

Die Breite des Regierungslagers in der Abgeordnetenkammer darf freilich nicht darüber hinwegtäuschen, daß die Union nationale auf ihrem linksliberalen Flügel eine sehr brüchige Angelegenheit blieb. Sowohl der Parti radical als auch der Parti républicain-socialiste et socialiste-français gerieten infolge des Kabinettseintritts von Spitzenpolitikern und der von großen Teilen in Partei und Fraktion vollzogenen Neuorientierung in heftigste innere Turbulenzen, auf die hier im einzelnen nicht eingegangen zu werden braucht ${ }^{89}$. Das Werk der "reconstruction“ und Disziplinierung, das Herriot in den Jahren nach dem Ersten Weltkrieg als Vorsitzender des Parti radical umsichtig betrieben hatte, ging dabei, nachdem es durch die zurückliegenden Entwicklungen bereits schwer angeschlagen war, vollends in die Brüche. Herriot selbst, der zwischen die innerparteilichen Fronten geriet, war als Vorsitzender nicht mehr zu halten und wurde im Oktober auf dem Parteikongreß von Bordeaux durch Maurice Sarraut abgelöst. In der Abgeordnetenkammer schlug sich die Aktivität einer weiterhin nach links orientierten Minderheit der Radicaux in einem oppositionellen Abstimmungsverhalten nieder. Im Herbst 1926 wurde auf Initiative von Léon Meyer sogar kurzzeitig der Versuch unter-

87 Ein ökonomisches Zeichen war am 20.12. 1926 die Intervention der Banque de France durch Dcvisenkäufe, um ein weiteres Ansteigen des Franc-Kurses zu verhindern. Vgl. Bonnefous, Histoire 4, S. 182.

88 Nach ebd., S. 182-184. Zur finanztechnischen Diskussion vgl. Blancheton, Le Pape et l'Empereur, S. $396-412$.

89 Ein Teil des Parti radical wollte Herriot sofort stürzen, ein anderer wollte ein neues Kartell nach der rentrée parlementaire. Diese inneren Probleme verbanden sich mit Widerständen gegen die Verwaltungsreform. Vgl. v.a. Polizeibericht vom 14. 9. 1926, „A.S. du Congrès Radical - Socialiste et de ses répercussions politiques“; AN Paris, F7 13191. Vgl. auch Berstein, Histoire 2, S. 18-22. 
nommen, eine separate radikal-sozialistische Fraktion zu gründen 90 . Bei den $R e^{-}$ publicains-socialistes stand am Ende nach heftigen internen Konflikten 1928 erneut die Spaltung in zwei rivalisierende Parteien ${ }^{91}$.

Nachdem der Sieg des Cartel des Gauches den Regierungsantritt eines Parteienbündnisses gebracht hatte, bedeutete die Union nationale von 1926 genau das Gegenteil. Das neue Regierungslager umfaßte jenes Mitte-rechts-Spektrum, das nur lose mit den organisationsschwachen "Parteien“ der Alliance démocratique und der Fédération républicaine verbunden war, aber auch einen beachtlichen Teil der beiden linksliberalen Parteien. Die Herstellung der Union war zudem, wie oben geschildert, durch individuelle Entscheidungen, vor allem jene Herriots, und nicht durch Parteivereinbarungen erfolgt. Poincaré suchte in der Folge den breiten Charakter seines Regierungslagers und seinen eigenen überparteilichen Status immer wieder zu betonen, indem er bewußt auch die Nähe zu Positionen des Parti radical suchte ${ }^{92}$.

Erstmals nach zwei Jahren der Regierungsstützung und des anschließenden Schwankens zwischen Regierungslager und Opposition stand die Kammerfraktion der SFIO jetzt wieder klar auf oppositionellem Terrain. Wie gesehen, nahmen die Sozialisten diese alte Rolle sofort energisch an und ließen den Kommunisten wenig Raum, sich parlamentarisch als Gegenkraft zur Union nationale zu profilieren. Fortan trat die SFIO in der Abgeordnetenkammer als große linke Oppositionskraft auf. Dabei wurde vor allem die Art und Weise einer Haushaltssanierung kritisiert, die auf indirekte Steuern statt auf eine Kapitalabgabe setzte ${ }^{93}$. Gleichzeitig entspannte sich die innerparteiliche Situation, die in den zurückliegenden Jahren durch Konflikte zwischen Anhängern und Gegnern einer Regierungsbeteiligung und zuletzt auch durch die ungewohnte Zersplitterung der Fraktion im Stimmverhalten gekennzeichnet war ${ }^{94}$. Die parlamentarische Alternativfunktion, die 1924-26 von den Mitte-rechts-Kräften auf traditionell diffuse Weise ausgefüllt worden war, gewann nun, infolge der Existenz einer systemloyalen und relativ modernen Oppositionspartei wieder ein klareres Profil ${ }^{95}$. Ähnlich wie in der Anfangsphase des Bloc national erschien die SFIO auch als möglicher Kern eines erneuerten Linksbündnisses. Neben dem linken Flügel des Parti radical rückten dabei für die Sozialisten - trotz demonstrativer Abgrenzung - allmählich auch die Kommunisten als eventuelle Partner ins Blickfeld ${ }^{9}$.

90 Bis 1913, als die Groupe radical et radical-socialiste geschaffen wurde, hatte es in der Kammer zwei Fraktionen des Parti radical gegeben: eine Groupe radical und cine Groupe radical-socialiste. Zur Ankündigung einer neuen Fraktion der Gauche radicale-socialiste vgl. Berstein, Histoire 2, S. $30 \mathrm{f}$.

91 Vgl. Billard, Parti républicain-socialiste, S. 414-427. Erst im Mai 1926 hatte die Fusion von Parti socialiste français und Parti républicain socialiste stattgefunden. Bei der Spaltung von 1928, der ein Jahr zuvor ein tumultuöser Parteitag vorausgegangen war, entstand neben dem Parti républicainsocialiste et socialiste français erneut ein Parti républicain-socialiste.

92 Vgl. Berstein, Histoire 2, S. 31-34.

93 Zur Kritik an Poincarés Haushalts- und Stabilisierungspolitik vgl. v.a. Ziebura, Blum, S. 272-278.

94 Judt, Reconstruction, S. 195, spricht davon, daß der Wechsel in die Opposition eine innerparteiliche "crise majeure" verhindert habe.

95 Daß Ziebura, Blum, S. 272, hier von einer "heikle[n] und undankbare[n] Aufgabe“ der SFIO spricht, erscheint - auch mit Blick auf das Wahlergebnis von 1928 - wenig begründet.

96 Ansätze zur Kooperation gab es bei den Senatswahlen vom 9. 1. 1927. Vgl. Bonnefous, Histoire 4, S. $201 \mathrm{f}$. Zum grundsätzlichen Kurs Blums gegenüber den Kommunisten im Jahr 1927 vgl. Ziebura, 


\section{Resümee: Triumph des Retters - Triumph der Tradition}

Innerhalb weniger Monate wurde Raymond Poincaré in der dominierenden öffentlichen Sichtweise vom "präsumptiven" ${ }^{97}$ zum tatsächlichen Retter Frankreichs. Erneut sei hier eine Analyse des deutschen Botschafters angeführt. In einem Bericht vom 5. November 1926 - kurz vor Ende der parlamentarischen Sommer- und Herbstpause - zog Hoesch ein Zwischenresümee der bisherigen Regierungszeit ${ }^{98}$. Die Kursentwicklung des Franc habe alle Hoffnungen übertroffen. Während man Poincaré zunächst "weithin mit Skepsis" begegnet sei, habe dieser nun ,im ganzen Lande außerordentlich stark an Ansehen gewonnen“. „Man hat", so Hoesch weiter, „im Lande das Gefühl, daß nach unaufhörlichen Schwankungen und ewiger Unsicherheit endlich ein Mann die Zügel der Regierung ergriffen hat, der wirklich regiert und weiß, was er will." Die breite Beliebtheit des Regierungschefs zeige sich unter anderem „in zahlreichen Karikaturen, auf denen Poincaré als der Beherrscher der Situation in irgend einer Form erscheint, in Annoncen oder Reklamebildern, die gleichfalls in irgend einer Form die Gestalt des Ministerpräsidenten verwenden, oder endlich in Revuen oder Kabarettliedern, in denen ,Raymond' als der energische Retter aus der Valutanot gepriesen wird“.

Die unverkennbaren Zeichen eines Personenkultes um Poincaré spiegeln eine extreme Personalisierung in der Wahrnehmung der Krisenüberwindung. Ganz falsch war diese Sicht freilich nicht, denn Poincaré befand sich in der Tat in der Rolle des tatkräftigen, die bisherigen parlamentarischen Blockaden und Wirrnisse souverän auflösenden Ministerpräsidenten. Erstmals konnte der erfahrene Politiker nun sein ansatzweise bereits 1922-24 praktiziertes Parlamentarismus- und Regierungsideal weitgehend umsetzen: Ein starker und auch mit populistischem Geschick auftretender Président $d u$ conseil über den Parteien, der nicht als parlamentarischer Mehrheitsführer, sondern als Patriot und Republikaner agiert, sowie eine breite, allen Maßnahmen parlamentarischer „Rationalisierung“ zugängliche Mehrheit der Abgeordnetenkammer. Die traditionelle, parteiunabhängige Ausfüllung der regierungstragenden Funktion verband sich in diesem „System Poincaré“ mit neuartigen Ansätzen parlamentarischer Disziplinierung zugunsten des quasipräsidentiellen Kabinettschefs. Diese Modifizierungen hatten allerdings - wie gerade der Vergleich mit der schroffen Forderung nach pleins pouvoirs seitens der vorhergehenden Regierung Briand-Caillaux zeigt - einen durchaus gemäßigten Charakter, und die gesamte Haltung war von Respekt vor den traditionellen Verfahrensweisen geprägt ${ }^{99}$. Anders als in der Endphase der deutschen Inflationskrise blieb das parlamentarische System intakt.

Blum, S. 374 f. Die kommunistische Strategie „Klasse gegen Klasse“ bildete aber noch jahrelang ein scherwiegendes Hindernis für eine Kooperation mit der SFIO.

97 Vgl. oben S. 504 die Bewertung des deutschen Botschafters.

98 Botschaftsbericht-Paris (Hoesch), A 3395, 5. 11. 1926, S. 1f.; PA AA Berlin, R 70717. Zur wachsenden Popularität Poincarés vgl. auch Bernard, La dérive des modérés, S. $319 \mathrm{f}$. Keiger, Poincaré, und Roth, Poincaré, gehen auf diese Thematik nicht ein.

99 Vgl. bereits Siegfried, Tableau des partis, S. 140, zu Poincaré: „Son génie fut de comprendre, mieux, de sentir qu'il fallait sauver le pays sans renier le régime“. 
Während Poincarés Ministerpräsidentschaft zur Zeit des Bloc national war dieses Regierungsverständnis nur mit großen Mühen umzusetzen gewesen. Der allmähliche Übergang der Radicaux in die Kammeropposition, die Forderungen auf der parlamentarischen Rechten nach einem klar definierten Regierungslager und nach der Führung dieses Lagers durch den Ministerpräsidenten sowie die Konkurrenz des präsidentiellen Führungsanspruchs Millerands hatten die Spielräume stark verengt. Poincaré war dabei zeitweise, besonders im Frühjahr 1924 bei der schwierigen Durchsetzung von double-décime und parlamentarischer Ermächtigung, eher ein mit Drohungen und List agierender Zuchtmeister als ein wirklich starker Ministerpräsident.

Zwar stand auch jetzt nicht die gesamte radikale Fraktion hinter der Regierung, und auch jetzt gab es Stimmen auf der Rechten, die mit dem überparteilichen Ansatz unzufrieden waren. Doch Poincaré befand sich nun in einer weitaus stärkeren Position: Der Parti radical war nicht mehr jene aufstrebende Kraft der Jahre 1923/ 24, die sich mehrheitlich aus dem nationalen Regierungsbündnis lösen wollte, sondern eine desillusionierte, zerstrittene Partei, deren Führung in das Kabinett eingebunden war und in der nur noch eine Minderheit an der Hoffnung auf ein erneuertes Linksbündnis festhielt. Gleichzeitig machte der glänzende währungspolitische Erfolg Poincarés die Kritiker auf der Rechten weitgehend mundtot. Die aus den Wahlen vom Mai 1924 hervorgegangene Abgeordnetenkammer fügte sich unter diesen Umständen erstaunlich ruhig in die ihr nun zugedachte Rolle. Der Triumph des Retters Poincaré war so auch der Triumph von dessen Regierungsund Parlamentarismusverständnis. An eine Grenze stieß dieser Ansatz dennoch. Die SFIO verweigerte sich dem angebotenen Eintritt in die Regierung der Union nationale und positionierte sich in der Abgeordnetenkammer durchaus energisch in der Opposition.

Die von Poincaré praktizierte Regierungsweise ist aus der Sicht einer funktionalen Parlamentarismusanalyse zunächst keineswegs negativ zu beurteilen: Die schwere Krise der regierungstragenden und damit auch der legislativen Funktion war überwunden, und die legislative Delegation vom August 1926 hielt sich in einem engen Rahmen. Aber auch in den übrigen parlamentarischen Grundaufgaben zeigte sich die Abgeordnetenkammer leistungsfähig: Die Alternativfunktion war voll gewährleistet, und die Beschränkungen des traditionellen parlamentarischen Kontrollinstrumentariums lassen sich auch als Anpassung an die modernen Realitäten interpretieren. Gleichzeitig verstummte die zuletzt grassierende Parlamentarismuskritik, und die erste Welle rechtsradikal-faschistischer Bewegungen in Frankreich ebbte bald wieder ab 100 .

Dennoch stellen sich bei distanzierter Betrachtung erhebliche Zweifel ein, ob der französische Parlamentarismus 1926 eine positive Weichenstellung erlebt hat. Nach dem Scheitern einer präsidentiellen Wende 1923/24 und nach dem Debakel eines neuartigen Modells der regierungstragenden Funktion in einem koalitionsähnlichen Linksbündnis triumphierte jetzt ein Parlamentarismusmodell, das weiterhin einem traditionellen Dualismus von Parlament und Regierung verhaftet

100 Vgl. v.a. die detaillierte Darstellung bei Douglas, From Fascism, S. 125-146. Speziell zum Problem, das die große Beliebtheit Poincarés für die rechtsradikalen Bewegungen aufwarf, ebd., S. $125 \mathrm{f}$. 
blieb. In der von Poincaré praktizierten Form war es kaum erfolgreich auf spätere Konstellationen übertragbar, denn es lebte von situationsbedingten Faktoren: vom erneuten Niedergang des Parti radical sowie von den individuellen Qualitäten und nicht zuletzt wohl auch vom finanzpolitischen Glück des Ministerpräsidenten. Als problematisch läßt sich im Rückblick schließlich auch Poincarés Prestige als triumphierender Retter bewerten. Indem es dem Regierungschef anscheinend mühelos gelang, den Verfall des Franc zu stoppen und die parlamentarischen Funktionsprobleme zurücktreten zu lassen, erfuhr - dies sei hier als Hypothese formuliert - das in der französischen Geschichte immer wiederkehrende Phänomen einer übermächtigen Persönlichkeit ${ }^{101}$, gegen die sich das republikanische Milieu der Dritten Republik stets so sorgsam abgeschottet hatte, eine nachhaltig positive Bestätigung. Vermutlich konnte dies, so die paradoxe Ironie jener Entwicklung, nur einem untadligen Republikaner wie Poincaré gelingen.

101 Die Rolle Poincarés wurde in diesem Kontext bislang offenbar kaum beachtet. Eine Andeutung findet sich bei Gildea, The Past in French History, S. 78. Ebd., S. 62-111, auch allgemein zum bonapartistischen Mythos in der französischen Geschichte. 